\title{
Design and validation of a double-burden mid-upper arm circumference (MUAC) tape for South African children aged six to 24 months
}

\author{
S. McLaren ${ }^{1}$, L. Steenkamp ${ }^{2}$ and H.D. McCarthy ${ }^{1}$ \\ ${ }^{1}$ School of Human Sciences, London Metropolitan University, London, UK and \\ ${ }^{2}$ HIV and AIDS Research Unit, Nelson Mandela University, Gqeberha, South Africa
}

The nutritional double burden refers to a concurrent high prevalence of under- and overnutrition ${ }^{(1)}$.

Mid-upper arm circumference (MUAC) (measured using a tape) and weight-for-height (WHZ) are used to identify malnourished children ${ }^{(2)}$. However, current WHO MUAC and WHZ cut-offs show limited levels of agreement among different childhood populations ${ }^{(3)}$, including South African children ${ }^{(4)}$.

Therefore, alternative MUAC cut-offs have been proposed, achieving improved agreement ${ }^{(5)}$. Additionally, there is current interest in MUAC as a screening tool for overweight and obesity ${ }^{(6)}$. Equipment and personnel in resource-poor environments are shared between situations of under- and over-nutrition. Therefore, the aim of this study was to validate alternative MUAC cut-offs for South African children aged between six and 24 months, with a view to producing a single MUAC tape capable of identifying both under- and overnutrition.

Data from 348 young children were collected from community clinics and creches. Ethics approval (ref. no H15-HEA-DIET-003) and gatekeepers' permission was obtained. Using $\mathrm{WHZ}$ categories defined by $\mathrm{WHO}^{(2)}$ as the reference, MUAC cut-offs were proposed to define categories of nutritional status. Sensitivity, specificity, positive predictive value (PPV), negative predictive values (NPV) were calculated. The association between MUAC category and WHZ category allocation defined by the proposed MUAC cut-off values was assessed using a chi-square test, with Cramer's $V$ to determine the strength of the association and a Kappa test to determine the level of agreement between the two indicators. Analyses were conducted using SPSS v25.

Mean age was 13.57 months (SD, 5.34) and $49.8 \%$ of the sample was male. Mean MUAC $=15.32 \mathrm{~cm}(\mathrm{SD}, 1.04)$ and mean WHZ $=$ 0.50 (SD, 0.99). WHZ ranged from -3.2 to 4.57 .

MUAC $<13.0 \mathrm{~cm}$ had a sensitivity of $100 \%$ and specificity of $99.1 \%(\mathrm{PPV}=0.25, \mathrm{NPV}=1.0)$ for $\mathrm{WHZ}<-3$. MUAC between 13.1 and $13.8 \mathrm{~cm}$ had a sensitivity of $100 \%$ and specificity of $95.6 \%(\mathrm{PPV}=0.117, \mathrm{NVP}=1.0)$ for $\mathrm{WHZ}<-2$. MUAC between 16.5 and $16.9 \mathrm{~cm}$ had a sensitivity of $11.1 \%$ and specificity of $86.8 \%(\mathrm{PPV}=0.111, \mathrm{NPV}=0.868)$ for $\mathrm{WHZ}>+2 . \mathrm{MUAC}>17.0 \mathrm{~cm}$ had sensitivity of $90.9 \%$ and specificity of $80.1 \%(\mathrm{PPV}=0.235, \mathrm{NPV}=0.992)$ for $\mathrm{WHZ}>+3$. There was a significant agreement between MUAC and WHZ (Kappa $=0.255 ; \mathrm{p}<0.001$ ). There was a significant association between the proposed MUAC cut-offs and WHZ categories $($ Pearson Chi square $=233.52 ; \mathrm{df}=16 ; \mathrm{n}=348 ; \mathrm{p}<0.001)$. This association is strong (Cramer's $\mathrm{V}=0.410 ; \mathrm{p}<0.001$ ).

The double-burden MUAC tape proposed has potential for reducing resource requirements for screening for both under- and overnutrition in a South African context. Despite the poor performance of the tool for identifying overweight, it has a high sensitivity and specificity for acute malnutrition and obesity. Further research is warranted using a larger independent sample with a range of states of nutrition as well as measures of clinical outcomes to substantiate these preliminary results.

\section{References}

1. UNICEF, WHO, WBG (2021)

2. WHO (2013)

3. Grellety E, Golden MH (2016) BMC Nutr 2, 10

4. McLaren SW, et al. (2017) S Afr J Clin Nutr 30(4), 118-119.

5. Tessema M, et al. (2020) PLoS ONE 15(4), e0230502.

6. Talma H, et al. (2019) Arch Dis Child 104, 159-165. 